\title{
Notas para la configuración del vínculo entre libertad de cátedra y religión
}

\author{
Notes to Configurate the Bond between Academic \\ Freedom and Religion
}

\author{
Raúl MADRID \\ Pontificia Universidad Católica de Chile (Chile) \\ rmadrid@uc.cl
}

\begin{abstract}
Resumen: El presente trabajo tiene por objeto analizar las relaciones que se presentan entre la noción de libertad de cátedra y la religión, tanto desde el punto de vista del núcleo dogmático del mencionado derecho subjetivo, como en la perspectiva de sus relaciones históricas y del surgimiento del derecho moderno a la libertad de cátedra. Este análisis se realiza en el contexto de la universidad alemana, que contrasta fuertemente con la universidad escolástica, pero también en su posterior desarrollo mediante la universidad norteamericana. Este proceso culmina con la posición de la AAUP en 1915, que configura el estatus actual de las relaciones entre los centros universitarios laicos y confesionales. La tesis del artículo es que, si bien para la modernidad religión y libertad de cátedra parecen nociones excluyentes, en realidad la esencia misma de la garantía se configura por relación con la fe, ya sea de manera negativa o positiva.
\end{abstract}

Palabras clave: libertad de cátedra; religión; fe; universidad; ciencia.
Abstract: This paper aims to analyze the bonds that occur between the notion of academic freedom and religion, from the scope of the dogmatic core of the right, but in the line of their historical relations and the emergence of modern academic freedom as well. The examination is performed in the context of german research university, which strongly contrast with scholastic period, but also in the context of its further development in the American university. This process ends with the AAUP 1915 Declaration, which shapes the present status of relationships between denominational and public universities. The main proposal of the paper is that academic freedom and faith, despite they seem opposed to modern mentality, actually are inseparable at the essence of the right, either from a negative or a positive point of view.

Keywords: academic freedom; religion; faith; university; science.

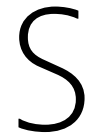

e suele argumentar que la libertad de cátedra es incompatible con la obligación de los académicos de respetar un ideario confesional, religioso, debiéndose igualmente por lo tanto aplicárseles la noción secular de libertad académica ${ }^{1}$. Esta diferencia insalvable estaría constituida por el siguiente razonamiento: (a) la religión (en realidad, la fe) es el acto por el cual

* Este trabajo forma parte del Proyecto FONDECYT n ${ }^{\circ} 114143$, titulado «El derecho a la libertad de cátedra y la evolución del concepto de universidad. Tres modelos para una misma garantía», en el que el autor se desempeñó como investigador principal.

1 Cfr. por ejemplo Curran, C., «Academic Freedom: The Catholic University and Catholic Theology», The Furrow, vol. 66, no 3 (1979), pp. 743 ss. 
se presta conformidad intelectual a un determinado cuerpo de proposiciones metafísicas y morales no por el valor intrínseco de su carácter veritativo, sino por la autoridad de quien lo enuncia, que en el caso de Dios no puede engañarse ni engañarnos ${ }^{2}$. Desde el punto de vista universitario, esto significaría la creación y mantenimiento de centros académicos cuyo punto de partida intelectual estaría basado en una verdad anterior al proceso mismo del conocimiento, es decir, a la aplicación de una cierta metodología, lo que determinaría la existencia de una ortodoxia a priori, que impediría el libre desarrollo de las ideas; (b) La libertad de cátedra sería una garantía de autonomía y tolerancia obtenida a costa de grandes sacrificios, desde los «oscuros» tiempos medievales, justamente para permitir que la conciencia intelectual de los académicos pueda desarrollarse y expresarse sin estar restringida o cooptada por ortodoxia alguna. Así, finalmente; (c) la libertad de cátedra sería incompatible con la existencia de universidades cuyo punto de partida no fuera simplemente el uso de la razón natural como causa de todo el conocimiento con pretensión intersubjetiva. Esta conclusión se puede formular también en otros términos: se conculcaría la libertad académica, en sus especies de investigación y docencia, a los académicos que, perteneciendo a este tipo de universidades, se les limitara su propio espacio de discrecionalidad al confrontarlo con los principios de la institución a la que pertenecen, lo cual sería un contrasentido respecto de la idea misma de universidad, por ser una «ciencia falsa» ${ }^{3}$.

La garantía de libertad de cátedra, por lo tanto, se levantaría contra la esencia misma de las religiones, en la medida en que éstas tuvieran algún reclamo científico y fundaran universidades para razonar en comunidad sobre sus presupuestos comunes. Las religiones, con sus ortodoxias, se opondrían al núcleo mismo de la libertad de pensamiento, que sólo se produce en la conciencia libre del sujeto. Esta disyunción intrínseca se vería avalada por el desenvolvimiento de la historia de la cultura y de las universidades, en la medida -se argumenta- en que el desarrollo de los diversos centros de educación superior a lo largo del tiempo no sería otra cosa que la relación de cuánto ha debido luchar la humanidad pensante para librarse del prejuicio.

La tesis que vamos a sostener en este trabajo es que, en realidad, la fe teológica, definida como el conjunto de proposiciones trascendentes en las

2 Concilio Vaticano I, Constitución Dogmática sobre la fe católica. Cap. 3, De la Fe, Dz. 1789.

3 Voltaire, Diccionario Filosófico, en Obras completas, con prólogo de Víctor Hugo, vol. IV, Casa Editorial de M. Senent, Valencia, 1894, p. 811. 
que se cree por la autoridad de un depósito, y la religión, como el conjunto de respuestas del hombre hacia la divinidad, no sólo no son incompatibles con la idea de libertad académica, sino que, por el contrario, se exigen hasta el punto de que la última es completamente inimaginable sin la primera, lo que lleva a formular una unidad necesaria entre la actividad académica humana y la apertura a la trascendencia, entendida como un segmento del saber que no se puede ni se podrá nunca justificar en términos lógico-formales.

Para abordar lo anterior, hay básicamente dos perspectivas, una teórica, basada en principios, y otra histórica, que se sustenta en los hechos pasados. Ambas, como veremos, se entrelazan, pero pueden distinguirse formalmente con claridad, porque responden a criterios que coexisten sin confundirse. Nos referiremos a ellos por separado.

\section{La Fe y EL PeNSAMIENTO CiENTíFico}

Hay una enorme distancia entre la universidad escolástica y la universidad ilustrada o moderna. En términos contemporáneos, la misma que media entre universidades confesionales y las que se declaran laicas, o todavía más tajantemente, «neutrales», aunque ambos tipos coexistan. La diferencia es una diferencia de contenido filosófico, que entraña también, además de un cierto escenario gnoseológico primario, un devenir antropológico de tal naturaleza que implica la disolución de un modelo en otro, por su inherente y radical incompatibilidad.

El primer modelo (el escolástico-confesional) acepta y recibe con toda naturalidad la existencia de un diálogo cierto, intelectualmente productivo, entre el conocimiento que proviene de las verdades reveladas, y el que se origina en el uso de la razón natural ${ }^{4}$. Estima que la información proporcionada por la fe es conocimiento sobre la realidad -Dios, el mundo, el hombre-, y no simplemente literatura o resultado de la imaginación de personas desconocidas que vivieron en el pasado. La cuestión de por qué se cree en un cierto depósito de fe y no en otro está radicada en la voluntad, movida por la gracia, $y$ es un tema que excede las presentes notas. La pregunta relevante en este punto, sin embargo, es por qué cabría acoger como un hecho normal de la vida intelectual un conjunto de proposiciones aparentemente indemostrables. A su

4 Cfr. por ejemplo San Anselmo, Monologion. Proslogion, vol. I, Cerf, París, 1986, p. 7. 
turno, la respuesta a esta nueva pregunta se encuentra en el modo de concebir al hombre, y en el rango de conocimiento que se le está dispuesto a atribuir como propio. Así, para esta idea de universidad, la presencia del misterio en el horizonte intelectual aparece como un elemento no conflictivo, aunque, por su propia naturaleza, sea superior a la razón humana. Lo misterioso (es decir, aquello que no se puede justificar racionalmente sobre las bases en que opera la inteligencia del hombre, y por lo tanto sólo puede ser indicado o señalado) es concebido como una realidad más con la que hay que contar, y como tal, forma parte de la estructura cognitiva humana, aunque la razón no sea capaz de justificarlo por sus propios medios.

Esta perspectiva no sufre un colapso frente a las realidades inexplicables, porque su propia comprensión del mundo le lleva a dotarla de cierta humildad intelectual, que se refiere no sólo a los grandes misterios religiosos, sino también al principio metafísico mismo, la existencia, que podría ser calificado como el primer y más importante misterio en el orden natural, en la medida en que el hombre es incapaz de comprenderla de manera exhaustiva. De este modo, la concepción del conocimiento que surge de esta perspectiva es integral, abarca la fe como realidad ineludible, y la razón natural en colaboración con ella para alcanzar la verdad de un mundo que supera la comprensión humana. Ello representa una ampliación del concepto de razón, no restringida a la dimensión empírica, sino abierta a otras posibilidades cognoscitivas 5 .

Así también, el académico escolástico se concibe a sí mismo como un colaborador de esta verdad, en sintonía con la universidad y con toda la actividad docente y reflexiva de la Cristiandad. Es por esta razón que la idea de libertad de cátedra, tal como se formula en la Modernidad, no puede tener cabida en el mundo escolástico, porque para que exista va a ser necesario que se produzca un hiato entre el profesor y la institución, de tal manera que puedan entenderse como contrapuestos, en sentido dialéctico. De hecho, si se revisan los múltiples procesos de censura a los académicos medievales, no se encontrará ni uno solo en el que, para defenderse, el afectado reclamara un «espacio de libertad» propio, un ámbito de discrecionalidad semejante al que se encuentra en el núcleo dogmático de la garantía moderna de libertad de cátedra ${ }^{6}$. En

5 RatZinger, J., Fe y ciencia, un diálogo necesario, Sal Terrae, Santander, 2011, p. 31.

6 Thijssen, J. M. M. H., Censure and Heresy in the University of Paris. 1200-1400, University of Pennsylvania Press, Filadelfia, 1998, p. 91. 
este contexto, parece ser que la existencia de un derecho a la «libertad de cátedra» exige presupuestos metodológicos y epistemológicos distintos a los que sustentan las universidades confesionales, en las que el diálogo de la ciencia y la fe se produce de un modo transitivo ${ }^{7}$. La idea de un espacio absoluto de independencia del profesor responde a un planteamiento negativo frente a dicha transitividad, y se origina en una modificación del concepto mismo de universidad, que pasa básicamente por una reducción de la idea de verdad a la de verificación, es decir, una jibarización de la trascendencia en una mathesis universalis ${ }^{8}$. Es negativo en cuanto la libertad de cátedra aparece como la ausencia de elementos que importunen el ámbito de libertad del académico, en el mismo sentido en que Warren y Brandeis hablarán de la privacy como el derecho a no ser molestado (the right to be left alone), en la doctrina norteamericana del siglo XIX 9 . De hecho, hay un vínculo muy importante entre el derecho a la libertad de cátedra que desarrollan en Estados Unidos y la noción de la privacy.

La nueva noción corresponde también a una modificación en el paradigma epistemológico de la noción de universidad, en cuanto ésta ya no aparece en búsqueda de verdades morales universales -por la reducción del concepto de verdad-. Así, este ámbito del saber queda reducido más bien al espacio privado, vinculado con la conciencia subjetiva ${ }^{10}$.

Tanto en el caso de las universidades confesionales, en consecuencia, como en el de la llamada «universidad de investigación», la idea de la religión y de la fe aparecen como cruciales para la configuración del concepto de libertad de cátedra, ya sea para rechazar que exista tal cosa, como en el caso de una visión integral del conocimiento humano del tipo escolástico, ya para crearlo, como una defensa de la conciencia subjetiva ante la potestad de la autoridad hermenéutica de la Iglesia y el poder político del gobierno terrenal.

7 MADRID, R., «El concepto de libertas scholastica y el modelo metodológico de la universidad medieval», Revista de Estudios Histórico furídicos, no 38 (2016), pp. 367 ss.

8 Descartes estaba convencido, como lo explica en las Regulae, que el método de las matemáticas puede ser aplicado a todos los contenidos, y que, por lo tanto, ese modo matemático lleva a una ciencia única, la mathesis universalis. Cfr. DesCARTES, R., Reglas para la dirección del espíritu. Hemos usado la versión: Regulae ad directionem ingenii, vol. X, Adam, C. y Tannery, P. (eds.), Léopold Cerf, París, 1908, p. 360.

9 Warren, S. y BrandeIs, L., «The Right to Privacy», Harvard Law Review, vol. IV, no 5 (1890), pp. 193-220.

10 KRISTEller, P. O., «Philosophy and Humanism in Renaissance Perspective», en The Renaissance Image of Man and the World, Ohio State University Press, Ohio, 1966, p. 40. 


\section{TRAYECTORIA HISTÓRICA}

Cabe distinguir aquí tres etapas en la relación del concepto de libertad de cátedra y la religión: el ámbito alemán, el ámbito norteamericano, desde la fundación del College hasta 1915, y desde tal fecha en adelante.

\subsection{La Lehrfreiheit»}

Es sabido que la libertad de cátedra surge formalmente como un derecho de primera generación, dentro del contexto de las exigencias de moderación del poder político frente a las libertades del individuo. Si bien hay algunos indicios en Francia, durante el siglo XVIII, su origen concreto se encuentra en la universidad alemana ${ }^{11}$. En el contexto alemán posterior a la Reforma, el poder en las universidades operaba de modo diferente de lo que ocurría en el ámbito católico. En este último, si bien es verdad que la potestad civil descubrió tempranamente durante la Edad Media los beneficios que podían reportar los centros universitarios para efectos de sus intereses -formación de profesionales, gestación de cuadros administrativos de élite, etc.-, existía durante dicho tiempo algo que podría denominarse «espíritu de cooperación» entre el príncipe y la sede romana, en el sentido de un acuerdo tácito que unía a todos los hombres en la Cristiandad latina, a saber, la conciencia vital de la existencia de un gran fin común para todos, ya fuera personal o institucional, consistente en alcanzar la vida eterna y la Civitas Dei por sobre los bienes adventicios y terrenos -como se hacía notar a propósito de la unidad epistemológica de la Cristiandad-. El mundo alemán reformado no podía comprender esto del mismo modo, no sólo porque su integridad epistemológica era diferente, sino porque ya se había trizado en diversas sectas. El frenesí de la creación y consolidación de los estados nacionales contribuyó, además de la fragmentación religiosa ya mencionada, a producir una situación en el que los Länder y sus gobernantes luchaban fundamentalmente por asegurar su situación en el mundo terreno, sin tener que coordinarse con una única fe, y dispuestos por lo tanto a abdicar muchas veces de los grandes principios trascendentes que antes parecían el elemento principal de cualquier empresa, en favor de consecuencias prácticas,

11 Cfr. Fichte, J. G., Über die einzigmögliche Störung der Akademische Freiheit, Carl Winter's Universitätsbuchhandlung, Heidelberg, 1905, pp. 29 ss. 
ya fueran políticas o sociales, dados los tiempos políticamente turbulentos en los que se encontraban.

La expresión española «libertad de cátedra» es una traducción de la voz lebrfreiheit (o, más precisamente, Akademische Lehrfreibeit). No tiene nada que ver con la expresión medieval libertas scholastica, que se refería más bien al conjunto de privilegios gremiales que cabían a los miembros de la institución universitaria en ciudades como Bolonia o París, pero entre los cuales no se contaba ningún atributo o garantía de naturaleza académica. La Lehrfreibeit implicaba que el profesor universitario era libre de examinar hechos, y de comunicar sus conclusiones en clase o por escrito. Es decir, el concepto contenía tanto la libertad de investigación como la libertad de docencia, que constituyen desde entonces el núcleo de la garantía. Ambos atributos eran consecuencia lógica de una cuestión de orden filosófico: la concepción idealista de la ciencia (Wissenschaft) que da origen a la llamada «universidad humboldtiana» o «universidad de investigación», el cual se configuraba como algo inacabado ${ }^{12}$, que constituye al mismo tiempo el motivo del desarrollo y la posibilidad de plenitud del sujeto considerado como una conciencia singular. Así, la Lebrfreibeit representaba algo más que la simple condición material de estar autorizado o no a hacer o realizar una determinada acción; constituía más bien una atmósfera de aprobación genérica a la labor científica y docente del académico, una especie de conciencia de que esta actividad libre era el modo adecuado en que el espíritu humano requería desplegarse para poder alcanzar todas sus potencialidades y en su totalidad, sin la opresión de fuerzas externas. Junto a ello, la Lebrfreibeit era valiosa por su significado en cuanto símbolo patriótico, parte del renacer nacional alemán, incluso más allá de los Länder locales. La misma Universidad de Berlín, dedicada en sí misma a la libertad de cátedra, era el símbolo de este renacimiento del Reich ${ }^{13}$.

El núcleo de la cuestión jurídica, sobre la que recayó su dimensión filosófica, fue el creciente malestar de los profesores por ser considerados como empleados del Estado, en iguales condiciones que el resto de los funcionarios públicos. Los académicos reclamaron que, por su especial desempeño en el

12 Paulsen, F., The German Universities and University Studies, Charles Scribner's Sons, Nueva York, 1906, p. 228.

13 Metzger, W., Academic Freedom in the age of the university, Columbia University Press, Nueva York, 1961, p. 113. 
ámbito intelectual, su espacio de discrecionalidad administrativa debía ser mayor que el de sus colegas que no se ocupaban directamente del discernimiento. Esta línea de mando administrativo significaba, además, que los profesores podían ser despedidos en cualquier momento. La idea de racionalización burocrática de los centros universitarios fue impulsada por el concepto de universidad de investigación que se originó en la Universidad de Berlín, y durante el siglo XX se expandió a todo el mundo. En ella, lo visible y lo racional triunfan sobre lo oral y lo tradicional ${ }^{14}$, como consecuencia de la intención de medir, ordenar, clasificar y ofrecer la generación de conocimiento con estándares semejantes a los de un artículo de consumo. En alguna medida, la desteologización tuvo por efecto objetualizar el conocimiento, de tal modo que la actividad del profesor pasa a constituir una celdilla en un gran panal, que puede ser calibrada a través de la medición de su productividad, la excelencia en la docencia o un estilo de vida relativamente aceptable. Como dice Clark, para alcanzar el éxito, es necesario también adquirir fama, estar de moda, ser «original», acreditar algún grado de genialidad. Esto se convierte en un tipo novedoso de carisma académico, unido a la escritura para el aplauso y el reconocimiento. El académico moderno emerge del cultivo de este nuevo carisma ${ }^{15}$, sobre la base de la afirmación kantiana de que la teología, en algún momento considerada la «reina de las ciencias» (regina scientiarum), no sirve en realidad ni a la sociedad ni a la verdadera religión, a menos que se conforme primero con los dictados de la razón natural ${ }^{16}$.

La idea de Humboldt no era, sin embargo, que las universidades fueran completamente autónomas, como las escolásticas. Consideraba que el Estado, a través de la responsabilidad sobre la administración de las universidades, constituía una excelente garantía de la libertad académica y de investigación. Se trataba de una cuestión de naturaleza laica -como no podía ser de otra forma, dados los contenidos presentes en la configuración de la Wissenschaft-, que se basaba en la idea de que el Estado debía asegurar la libertad de investigación y de enseñanza sin interferencia de ninguna especie por parte principalmente de las iglesias, pero tampoco de otros grupos o instituciones existentes en la sociedad. Con este objetivo, el gobierno designaba los profesores, en ocasio-

14 Clark, W., Academic Charisma and the Origins of the Research University, The University of Chicago Press, Chicago, 2006, p. 3.

15 Ibid., pp. 3-4.

16 KanT, I., Der Streit der Fakultäten, 1798. Usamos aquí la versión española, El Conflicto de las Facultades (Roberto Aramayo, trad.), Tecnos, Madrid, 2000, p. XX. 
nes contra de la opinión de las facultades, y podía intervenir en los claustros con objeto de que dejaran de lado conductas de carácter político.

Los profesores no se consideraban simples espectadores del acontecer, sino más bien sus oráculos, a través de cuyo medio se expresaban verdades trascendentes ${ }^{17}$. Es famoso el apasionado y violento debate entre Rudolf Virchow y Ernst Häckel ${ }^{18}$, en medio de la conflagración por el evolucionismo ${ }^{19}$. Virchow, atacando un escrito anterior de Häckel, advierte sobre la total libertad de investigación y publicación que había en Alemania, y sostiene que la obligación de los hombres de ciencia, aunque sólo sea para mantener el estado de cosas, consiste en ser responsables de lo que dicen y escriben. Insiste en la necesidad de establecer una línea clara de demarcación entre las proposiciones que pueden ser consideradas verdades establecidas, y otras que no son más que mejor o peor fundadas especulaciones ${ }^{20}$. Es decir, es necesario distinguir entre hechos e hipótesis. El que se dirige al público -acota Virchow- tiene una doble obligación de confirmar la verdad de sus dichos (es decir, la obligación con el Wissenschaft, y la obligación clásica de no confundir a las audiencias con afirmaciones erróneas), y por lo tanto, las afirmaciones no probadas no pueden ser presentadas ante las audiencias como verdaderas ${ }^{21}$. Este discurso causó gran impacto en toda la comunidad científica europea, por cuanto fue considerado como «una promesa de próxima liberación de la tiranía del pseudodogmatismo filosófico que tanto había obstruido el progreso de la ciencia» ${ }^{22}$.

La respuesta de Häckel comienza diciendo que «sus puntos de vista (de Virchow) están en oposición tan fundamental con los míos, y rozan tan de cerca nuestras más queridas concepciones morales, que no hay reconciliación posible». Considera que no existe tal línea demarcatoria, porque todo conocimiento humano en cuanto tal es subjetivo. Una ciencia objetiva que consista

17 MetzGer, W., op. cit., p. 114.

18 Para esta discusión, cfr. ElaM, Ch., «Häckel and Virchow: the Evolution Controversy in Germany», The Contemporary Review (octubre de 1878), pp. 541-569.

19 Cfr. Huxley, T. H., «Nota Introductoria», en HäCKEL, E., Frei Wissenschaft und Frei Lebre, Stuttgart, 1878. Citamos aquí por la edición en lengua inglesa: Freedom in Science and Teaching, C. Kegan Paul \& Co., Londres, 1879, pp. IX ss.

20 Virchow, R., Die Freibeit des Wissenschaft im Modernen Staate, Berlin, 1877. Citamos aquí por la versión en lengua inglesa: Freedom of Science in the Modern State. A Discourse Delivered at the third General Meeting of the fiftieth Conference of the German Association of Naturalists and Physicians at Munich, on the 22nd of September, 1877, John Murray, Londres, 1878, p. 8.

21 Virchow, R., op. cit., pp. 23-4.

22 Elam, C., «Häckel and Virchow: the Evolution Controversy in Germany», The Contemporary Review (octubre de 1878), p. 544. 
sólo en hechos -argumenta-, sin ninguna teoría subjetiva, es inconcebible. Así, la idea de Virchow, según Häckel, de que existen opiniones de las que no se puede dudar sería equivalente al modelo del «infalibilismo» religioso. Como era de esperarse, casi todos los científicos alemanes adhirieron a esta última posición. Desde el punto de vista de la idea de la libertad de cátedra, pues, esta discusión tuvo por consecuencia reafirmar la distancia con una posición en que la verdad desempeñara un papel absoluto, $\mathrm{y}$, a contrario sensu, fortalecer la idea de tolerancia como base de la actividad académica, en cuyo ejercicio no se está atado a ninguna autoridad. Las conclusiones son del exclusivo juicio del profesor, y él responde por ellas. Su único objetivo es la verdad, es decir, la adecuación del pensamiento con la realidad, sin que intervenga autoridad externa alguna ${ }^{23}$.

Fuera de la universidad, ya no existía el mismo grado de libertad. La Lebrfreibeit no cubría las opiniones de los académicos en el ámbito externo, sobre materias políticas o sociales en general (la cuestión política era, en realidad, el campo que más problemas podía traer, por lo que se pensaba que era mejor que los académicos se mantuvieran al margen de ella, lo que no se lograba en los hechos). Como se entendía que los académicos eran funcionarios públicos, se esperaba de ellos, además, que dieran el ejemplo en lo relativo al resguardo de la ley y de los criterios cívicos. Metzger considera que esta separación entre la actividad interna y externa del académico era un reflejo de la dualidad filosófica alemana, a propósito de la diferencia kantiana entre el mundo de la heteronomía y el de la autonomía. La reducción de la libertad académica al ámbito interno de la universidad sugiere, dice Metzger, la formula luterana de la libertad espiritual combinada con la obediencia temporal ${ }^{24}$.

\subsection{La universidad norteamericana}

Una diferencia importante del sistema norteamericano, para los efectos que aquí interesan, fue la estructura de sus autoridades. La universidad colonial era administrada por un Presidente (Rector), además del Board o Consejo. Ambas figuras habían sido importadas de Europa, aunque no propiamente del

23 Paulsen, F., «Die Akademische Lehrfreiheit und ihre Grenzen: eine Rede pro domo», Preußische fabrbiicher, vol. XCI (1898), p. 517.

24 Metzger, W., op. cit., pp. 116-7. 
mundo de la organización académica, donde primaba la administración por el claustro. La autoridad final era ejercida por el Consejo, sin perjuicio de la potestad del Rector en la administración cotidiana. Así pues, la estructura de gobierno estaba compuesta por un cuerpo profesional e interno (el Rector y su equipo), y otro órgano de gobierno externo, integrado por personas ajenas al claustro. Este predominio del Consejo generaba molestia en la comunidad estrictamente académica, que aspiraba en principio a un tipo de gobierno más parecido al modelo tradicional europeo de autonomía universitaria.

Otra instancia de conflicto era la relación del college con la autoridad pública. Estas dificultades se produjeron primeramente con la Corona, que debía autorizar la creación de los centros educativos, lo que determinaba un control sobre su gestión que disgustaba a los administradores norteamericanos. Posteriormente, las desavenencias se reprodujeron con las autoridades locales, por cuanto no existía un gobierno central que estableciera conductas generales para todo el país en este tema, y en consecuencia era preciso remitirse a la administración de cada colonia para tomar las decisiones pertinentes, lo que era oportunidad de mayores desencuentros en la coordinación de los intereses de cada denominación religiosa. El problema de fondo era cómo operar de acuerdo con la legislación -en sentido amplio-, y al mismo tiempo conservar la autonomía institucional.

Desde el punto de vista de los objetivos educativos, los primeros colonizadores se veían a sí mismos como los responsables de asegurar la continuidad de la educación occidental en el nuevo continente, cuestión que consistía esencialmente -para ellos- en que la fe y sus asuntos podían ser abordados por la razón natural. Paralelamente a este objetivo, cada denominación religiosa (anglicanos, calvinistas, presbiterianos, etc.) necesitaba formar a sus respectivos clérigos, para poder asentarse sólidamente y ganar adeptos. Esto fue el elemento determinante en la fundación de los colleges coloniales: la tradición cristiano-reformada constituyó la piedra basal de la estructura educativa de Estados Unidos ${ }^{25}$. La Universidad de Harvard fue fundada en 1636 con el objeto de satisfacer estas metas, recibiendo a los hijos de los inmigrantes que habían estudiado en Oxford y Cambridge. Para sus primeros académicos, Harvard era la pica en Flandes de la militia universal de Cristo en los nuevos territorios. Las universidades, y especialmente esta última, tenían el cometido de separar

25 Brubacher, J. S. y Rudy, W., Higher Education in Transition. A History of American Colleges and Universities, Transaction Publishers, New Brunswick, 2008, p. 6. 
las aguas entre la civilización y la barbarie ${ }^{26}$. Así pues, la educación en Estados Unidos nace vinculada con la iglesia reformada, como un instrumento para su preservación y desarrollo, lo que suponía también, de modo muy relevante, la formación moral de sus habitantes.

Tal como ocurrió en su momento con la universidad escolástica, la mayoría de los profesores de la época colonial eran religiosos. La multiplicidad de credos, sin embargo, constituía la diferencia con el tiempo medieval católico -si hemos de seguir la comparación-. La orientación de la universidad colonial norteamericana era, en consecuencia, abiertamente religiosa, pero la ausencia de unidad en la fe de la población generó en primer lugar una rivalidad entre las diferentes congregaciones, junto con cierto espíritu de tolerancia (que se manifestó, por ejemplo, en la eliminación de los exámenes teológicos para ingresar a las universidades, o el eclipse de la ortodoxia puritana en Harvard). Este espíritu de tolerancia propició a la postre la secularización del sistema universitario, porque la imposibilidad de entrar en las cuestiones teológicas de fondo, preteridas con objeto de privilegiar los acuerdos de conveniencia, fue poco a poco silenciando la discusión dogmática de carácter público, en favor de alcanzar el acuerdo sobre problemas contingentes y, sobre todo, capaces de reportar consecuencias favorables a los intereses de cada grupo en particular, y haciendo necesaria poco a poco la garantía de libertad de cátedra.

Así, el siglo XVIII vio crecer esta diversidad religiosa cada vez menos diversa en la acción, caminando hacia su reducción civil en estructuras secularizadas. A mediados de este siglo, las ideas de la Ilustración y el racionalismo desembarcaron en las colonias norteamericanas, contribuyendo a cambiar la cultura dominante. Así también arribó el humanismo, que se oponía a la enseñanza de los estudios religiosos fuera de las facultades de teología. Muchos de los padres fundadores nacidos en la primera mitad del siglo XVIII, como Washington, Jefferson o Franklin, adhirieron al racionalismo y a las teorías de los derechos del hombre y el ciudadano, por lo cual se encontraban menos vinculados a la observancia religiosa organizada; incluso no consideraban a la Biblia como un texto revelado ${ }^{27}$. Esta actitud se reflejó en la organización de

26 Rudolph, F., The American College \& University: A History, The University of Georgia Press, Athens, 1990, p. 6.

27 Cohen, A. M. y Kisker, C. B., The Shaping of American Higher Education: Emergence and Growth of the Contemporary System, Jossey-Bass, San Francisco, 2010, p. 17. 
los colleges en al menos dos aspectos: (a) la composición del Consejo, como fue el caso de Harvard en 1727, que pasó a estar integrado por algunos hombres de negocios de Boston -completamente ajenos al claustro, pero vinculados al mundo práctico-, y (b) el fortalecimiento de la enseñanza de las profesiones seculares, en virtud de que el número de alumnos para la carrera eclesial había disminuido significativamente, como fue el caso de Yale (fundada en 1701). En este sentido, como dice Rudolph ${ }^{28}$, las universidades desempeñaron el papel de forjar un sentido de unidad que la religión reformada no conseguía producir, por cuanto el sustrato de la población incluía diversas nacionalidades y credos, y así enseñarles a todos por igual el manejo de las cosas del mundo. Hubo una reacción contra este iluminismo del siglo XVIII, conocida como The Great Awakening, a partir de 1750, reapareciendo varias veces a lo largo del tiempo, bajo distintas formas. Al final del período colonial, la diversidad y la tolerancia se habían convertido, sin embargo, en valores de tal efervescencia cultural, que los colleges conservaban poco interés en la religión, y permanecían vinculadas a algún credo sólo de un modo muy formal y poco exigente con sus propios principios.

Así, crece la idea de la libertad como liberación del yugo impuesto por las denominaciones religiosas que desempeñaban un papel educativo, y que ejercían de hecho un poder enérgico sobre el sistema. Hay una diferencia entre la «liberación» que aconteció en su momento durante el Renacimiento, respecto de la Iglesia Católica, y el combate de los liberales contra las iglesias y credos presentes en Estados Unidos. La discusión europea, si bien es verdad que tiene una dimensión práctica, político-administrativa, era fundamentalmente teórica, y radicaba en la discusión sobre si cabe aceptar o no una autoridad dogmática que al mismo tiempo fuera secular. El conflicto en Estados Unidos tiene un cariz distinto, porque se plantea contra una simplificación de la autoridad dogmática en sentido filosófico, que escondía en realidad una lucha de poder. Las iglesias que desembarcan en Norteamérica son todas protestantes, y por lo tanto traen consigo el elemento opuesto a una autoridad central, ya sea hermenéutica o política. En consecuencia, ese afán de dominación no podía estar regido por un principio distinto que el de la expansión indefinida, sin que ello implicara necesariamente una reflexión teológica de gran calado intelectual. Esta característica produce un especial rechazo hacia las instituciones universitarias de inspiración religiosa, que se afianza -como hemos visto- a lo largo

28 RUdOLPH, F., op. cit., p. 13. 
de la historia del College, y durante el período de su conversión a la Research University. Este es el contexto en el que se formula la idea de libertad de cátedra que hoy resulta dominante.

\subsection{La «Declaración» de 1915}

A partir de la década de 1880 , un conjunto de casos relativos a la libertad de cátedra afectaron la profesionalización de las ciencias sociales norteamericanas. Tales casos fueron presentados en su momento como censura a la libertad de los miembros de la profesión académica por parte de los Consejos y de ciertos políticos conservadores, lo que produjo una reacción de parte de los profesores «liberales»: se formaron espontáneamente grupos reducidos de destacados académicos que, en los hechos, determinaban quién sería defendido y cómo en cada universidad, dependiendo muchas veces de su filiación o inclinación política. De esta manera, los debates concretos sobre la libertad de cátedra ayudaron a configurar los límites del disenso permitido por los propios colegas, dentro de la naciente clase de académicos profesionales. Una cierta «ortodoxia» comenzó, por lo tanto, a extenderse por las universidades (conservadora o liberal, según el caso) ${ }^{29}$, que derivó finalmente en la redacción del texto de 1915, el cual manifiesta un claro predominio del área «liberal». El tenor era una mayoría de profesores «progresistas» que se oponían a los Consejos «conservadores», de tal manera que los supuestos casos de violación de la libertad de cátedra sirvieron para ir señalando los límites de lo que la mayoría estaba dispuesta a aceptar en el área de las ciencias sociales, creando en cierta forma, o poniendo las bases, para que el instrumento jurídico de la libertad de cátedra pudiera ser utilizado de hecho para amedrentar a la facción políticamente contraria, y conseguir de ese modo la hegemonía al interior de las facultades, desvinculando a los académicos de otras tendencias. Este era el estado de cosas a la altura de la formación de la American Association of Universitary Professors (AAUP). La Declaration de 1915 traduce estas ideas en la noción de libertad de cátedra. John Dewey (1859-1952), uno de los fundadores de la asociación, era una de las figuras más importantes de la llamada «pedagogía progresista», cuyos

29 Furner, M. O., Advocacy \& Objectivity. A Crisis in the Profesionalization of American Social Science, 1865-1905, The University Press of Kentucky, Lexington, 1975, p. 8. 
objetivos volverán a ser planteados durante las revueltas universitarias de la década de los sesenta en el siglo XX.

Cuando Edward Ross fue despedido de Stanford en 1900, por sus desacuerdos con Jane Stanford, Arthur Lovejoy (1873-1962), profesor de filosofía de Johns Hopkins consideró que el problema de la dependencia de los profesores respecto de los Consejos y los donantes tenía que encontrar un camino de solución. En enero de 1915, Seligman, Lovejoy y Dewey, junto a otros destacados académicos, fundaron en Nueva York la AAUP, siendo Dewey su primer presidente, y Lovejoy el primer secretario. En diciembre de ese mismo año, la AAUP publicó el primer ejemplar de su Bulletin of the American Association of University Professors, en el que se incluyó la Declaration, a cuyo contenido nos referiremos a continuación ${ }^{30}$.

La Declaration de 1915 fue la primera sistematización del concepto moderno de libertad de cátedra. Este documento ha servido de modelo en todo el mundo, de tal manera que la noción dominante de libertad de cátedra coincide hoy en día con su propuesta, y con las nociones desarrolladas por la doctrina norteamericana ${ }^{31}$. Como estableciera Metzger ${ }^{32}$, en el ámbito norteamericano es posible identificar dos concepciones de libertad de cátedra: la definición profesional, que fue formulada por primera vez por (AAUP) en 1915, y la definición constitucional, desarrollada por las sentencias de la Corte Suprema desde la década de 1950 hasta la fecha. En el primer caso, el objeto era proteger la libertad profesional de los académicos para investigar, enseñar y opinar sobre cualquier asunto, especialmente a los universitarios, como veremos al exponer la Declaración de 1915 y sus comentarios posteriores (es decir, se trata de la noción de «libertad de cátedra» propiamente tal). La perspectiva de la libertad constitucional, por su parte, hace referencia más bien a la autonomía universitaria $^{33}$.

30 Cfr. TiEde, H., University Reform: The Founding of the American Association of University Professors, Johns Hopkins University Press, Baltimore, 2015, pp. 59 ss.

31 Cfr. por ejemplo Academic Freedom: A Guide to the Literature, Greenwood Press, Westport, 2000. La mayoría de las entradas corresponde a doctrina norteamericana.

32 Metzger, W., «Profession and Constitution: Two definitions of Academic Freedom in America», Texas Law Review, vol. 66 (1988), p. 1265.

33 «No está claro cuánto protege la libertad de cátedra constitucional a los académicos considerados individualmente, o si, como la mayor parte de los fallos de los tribunales indican, ésta salvaguarda primeramente -y quizá únicamente- la libertad institucional de las universidades y los colleges», BARENDT, E. M., Academic Freedom and the Law. A Comparative Study, Hart Publishing, Oxford, 2010, p. 161. 
Prácticamente la totalidad de las definiciones de libertad de cátedra que se encuentran en la doctrina apuntan a la idea de la libertad «profesional», es decir, aquella relativa al profesor singular, que se enfrenta con poderes que dependen del Estado o los administradores de las universidades, y no a la autonomía institucional -que constituye un problema jurídicamente distinto- ${ }^{34}$. La idea de una libertad de cátedra profesional protegida constitucionalmente es controvertida, y carece, al menos de momento, de una aceptación general ${ }^{35}$, sin perjuicio de que la Corte Suprema de Estados Unidos afirmara que «nuestra Nación se encuentra profundamente comprometida con el resguardo de la libertad de cátedra, que es un valor trascendente para todos, y no sólo para los profesores afectados. Esta libertad es por lo tanto una preocupación especial de la Primera Enmienda, que no tolera leyes que extiendan la ortodoxia sobre las aulas» ${ }^{36}$.

La garantía de libertad de cátedra alcanzará su configuración a partir de casos concretos de conflicto entre profesores y administradores de las universidades, aproximadamente a partir de la última década del siglo XIX en ade-

34 Citamos algunas definiciones que demuestran lo anterior: «La 'libertad de cátedra' hace referencia a un conjunto de libertades técnicas: enseñar, averiguar (investigate), investigar (research) y publicar sobre cualquier materia, por un interés profesional, sin peligro en el ámbito académico (vocational jeopardy) o amenaza de cualquier otra sanción, salvo, únicamente, por una infracción de la ética profesional en el ejercicio de esta libertad, debidamente demostrada. Específicamente, lo que distingue a la libertad de cátedra como una libertad diferente es la reivindicación profesional de una responsabilidad especial y limitada, referida a todas las actividades académicas del profesor-investigador: una responsabilidad no referida a un parámetro institucional o social de beneficio económico, o un interés aceptable, o un pensamiento correcto o una teoría construida socialmente, sino exclusivamente a un parámetro fiduciario de integridad profesional». ALSTYNE, W. VAN, «The Specific Theory of Academic Freedom and the General Issue of Civil Liberty», The Concept of Academic Freedom, vol. 404 (1972), pp. 146-147; «La libertad de cátedra es la libertad del académico, considerado en cuanto individuo, en su enseñanza e investigación, para buscar la verdad donde quiera que ésta parezca conducirlo, sin miedo al castigo o a ser despedido por haber ofendido alguna ortodoxia política, religiosa o social». BERDAHL, R., «Universities and Government in the Twenty-First Century: The US Experience», in Towards a New Model of Governance for Universities? A Comparative View, Jessica Kingsley, London, 1999, p. 60 (segundo énfasis propio); «[la libertad de cátedra] le da a los investigadores y científicos la libertad de buscar y presentar la verdad, tal como la ven, a sus alumnos y al público en general, tanto de forma oral como de forma escrita, lo que es posible gracias a la ausencia de sanciones, que son impuestas por personas poderosas o grupos al interior del campus, o fuera de éstos». Berry. R., Academic Freedom and Peer Reviews of Research Proposals and Papers, American Fournal of Agricultural Economics, vol. 62, nº 4 (nov. 1980), p. 640. Y así, sucesivamente.

35 Wright, R. G., «The Emergence of First Amendment Academic Freedom», Nebraska Law Review, vol. 85 (2007), p. 793.

36 Keyishian v. Bd. Of Regents, 385 U.S. 589, 603 (1967). 
lante. Algunos ejemplos: Richard T. Ely, de la Universidad de Wisconsin, en 1894, fue acusado por «herejía sociológica» debido a que su postura en dichas materias no era coherente con los principios conservadores de su tiempo ${ }^{37}$. En el mismo año, a I. A. Hourwich de la Universidad de Chicago, se le pidió que optara entre dejar a un lado la política, o renunciar a su trabajo, por causa de haber participado en una Populist convention. El implicado se decidió por la última opción. El profesor E. W. Bemis fue despedido de la Universidad de Chicago en 1895, aparentemente por su oposición a ciertos monopolios privados $^{38}$. En 1897, el profesor James A. Smith fue despedido del College Marietta, en razón de sus enseñanzas anti monopolio ${ }^{39}$. Durante el mismo curso, el Rector E. B. Andrews -de la Universidad de Brown- renunció, luego de que se le pidiera que se abstuviera, o bien, que expresara su opinión favorable, a la libre acuñación de plata, pues su postura era contraria a la de los donantes que ayudaban al sustento económico de la Universidad ${ }^{40}$. El más conocido fue el caso del profesor de economía Edward A. Ross -ya mencionado antes-, quien

37 La denuncia fue hecha por Oliver E. Wells, quien fuera miembro del Consejo de Regentes de la Universidad de Wisconsin. Wells hizo su acusación al periódico, vinculando a Ely con las huelgas sufridas en Madison; la situación tiene la agravante de que, antes de la llegada de Ely, nunca hubo una huelga. De acuerdo con Wells, los principios del actuar de Ely están contenidos en su libro Socialismo: una revisión de sus fortalezas y debilidades, con sugerencias para una reforma social. Con posterioridad a dicha denuncia, el Consejo de Regentes constituyó un comité para investigar los hechos. Las pruebas no fueron suficientes para aplicarle una pena a Ely, de modo que quedó exonerado de todos los cargos. Cfr. Herfurth, T., Sifting and Winnowing, University of Wisconsin, Madison, 1949, disponible en http://digicoll.library.wisc.edu/WIReader/ WER1035-Chpt1.html\#Footback05 (16 de febrero de 2015).

38 En opinión de Bemis, éste fue despedido por consideración a los capitalistas, ya que en sus intervenciones públicas hablaba contra sus intereses. Lo cierto es que Bemis participaba en un movimiento que era contrario a que los servicios públicos fueran propiedad de sujetos privados. Sin embargo, de acuerdo con William R. Harper-Rector de la Universidad de Chicago-, Bemis fue despedido porque no era un profesor competente para seguir en su cargo. De ahí que se discuta si efectivamente este caso fue uno sobre libertad de cátedra o no. BOYER, J., Academic Freedom and the Modern University: The Experience of The University of Chicago, College of the University, Chicago, 2002, pp. 13-7.

39 James Smith comenzó sus actividades como profesor de economía y sociología en 1895. Según Beach, sus ideas eran visionarias, razón por la que el consejo de administración resolvió no renovar su contrato, puesto que los donantes que contribuían para pagar su remuneración, decidieron no continuar haciéndolo. A pesar de que los alumnos enviaron una petición formal, con el objeto de que el profesor se reincorporara a sus funciones, los miembros del consejo de administración se negaron a tramitar la solicitud; posteriormente, Smith fue contratado por la Universidad de Washington. BEACH, A. G., A Pioneer College. The History of Marietta, Impresión Privada, Chicago, 1935, pp. 226-7.

40 Cfr. Brubacher, J., y Rudy, W., op. cit., pp. 312-3. 
en 1900 fue forzado a dimitir de su cátedra en la Universidad de Stanford. Su desavenencia con la viuda del fundador, Jane Stanford, provenía del enfoque progresista del afectado. Stanford, a su vez, era presionada por sectores conservadores de California, a quienes disgustaban las afirmaciones de Ross ${ }^{41}$. La importancia de este caso radica en que (a) puso de manifiesto las discrepancias entre fundadores y donantes -por una parte-, y algunos profesores, por otra $^{42}$, y (b) porque el caso llamó la atención de todo el país, incluyendo a la Asociación Norteamericana de Economía, la cual constituyó un comité para investigar el despido de Ross, expidiendo en 1901 un informe favorable a este último. Ross no fue, sin embargo, reintegrado. Esta fue la primera ocasión en la historia universitaria norteamericana en que una tercera instancia tuvo conocimiento procesal y juzgó el despido de un académico ${ }^{43}$. Así, el debate sobre la libertad de cátedra se trasladó a la esfera pública, que adoptó básicamente tres posiciones: (a) los que consideraron que el problema era más bien aparente; (b) aquellos a los que la noción de libertad de cátedra les parecía un concepto anarquista, y (c) los que aceptaban la posibilidad de desarrollarla desde un punto de vista técnico-jurídico, aunque subordinada a la misión de la universidad, para así mantener la reputación de la institución de educación superior correspondiente ${ }^{44}$.

La Declaration of Principles of Academic Freedom and Academic Tenure de 1915 buscaba otorgar a los académicos una mayor protección, en el ámbito de la libertad de expresión, de las que consagraban las leyes de su tiempo. Defiende que la libertad de cátedra debe ser entendida como un resguardo de los profesores frente al despido y los castigos por este tipo de causas, es decir, la salvaguarda de la profesión académica concebida como un desempeño laboral en una institución de educación superior cuyo papel es significativo en el contexto de toda la sociedad: «Si la educación es la piedra angular de la estructura de la sociedad, y si el progreso del conocimiento científico resulta esencial para la civilización, pocas cosas serán más importantes que incrementar la dignidad de la profesión académica, con vistas a atraer hacia sus filas a los hombres de mayor capacidad, de sólido conocimiento y de carácter fuerte

41 Es interesante atender al punto de vista de Ross, que lo explica en el capítulo VII del libro autobiográfico Seventy Years of it: An Autobiography, Arno, 1977, pp. 64-86.

42 VEYSEY, L. R. The Emergence of the American University, The University of Chicago Press, Chicago, 1970, p. 400.

43 Brubacher, J., y Rudy, W., op. cit., p. 319.

44 VeYseY, L., op. cit., p. 407. 
e independiente» ${ }^{45}$. Por lo tanto, los académicos no son empleados, sino individuos seleccionados por los administradores, porque en cuanto elegidos, las autoridades carecen de competencia moral y del derecho para intervenir en su actividad, porque se trata de una profesión que tiene sus propios protocolos de rigor científico, y éstos deben ser respetados. En esta línea, el 1915 General Report incorporó un lenguaje destinado a destacar la naturaleza profesional del trabajo académico ${ }^{46}$. La configuración de este status en términos jurídicos viene a ser una especie de corolario de una tendencia iniciada largo tiempo antes, en la ilustración, que derivaba del auto-reconocimiento de los académicos como una casta independiente, en cierto modo superior al resto de la sociedad por causa del carácter técnico de su trabajo, y del servicio que prestan a ella.

El objetivo de los redactores era desestimar la idea recién mencionada de que los académicos constituían funcionarios a merced de la arbitraria tolerancia de sus empleadores. Con esta meta, los ejes sobre los que estructuraron la idea de libertad de cátedra fueron los siguientes: libertad de investigación, libertad de enseñanza y libertad de expresión fuera de los recintos universi$\operatorname{tarios}^{47}$. Tanto el texto de 1940, como los comentarios a éste realizados en la década de los años setenta, y la elaboración jurisprudencial ulterior, constituyen especificaciones o elaboraciones que se sustentan en la línea trazada por los profesores de 1915, mas no un cambio estructural en el concepto delineado a principios del siglo XX. El modo en que se concibe la libertad de cátedra es funcional: sin ella, el profesor no hace avanzar el conocimiento, ya sea a través de su alcance o de su difusión.

Por su parte, en el 1940 Statement of Principles de la AAUP se alcanzó un acuerdo con la Association of American Colleges, la principal organización de las Artes Liberales en los colleges (para el texto de 1915 no lograron concitar el apoyo de las universidades). El texto de este acuerdo es lo que ha fijado los parámetros de la libertad de cátedra profesional en Estados Unidos desde entonces $^{48}$, resumiendo de manera concisa las líneas generales de la libertad de cátedra formuladas en el texto de 1915 . Se reafirma el pleno derecho de los académicos para investigar y publicar los resultados de su investigación. Se

451915 Declaration of Principles on Academic Freedom. 2. The Nature of Academic Calling.

46 Seligman, E. R. A. et al., «General Report of the Committee on Academic Freedom and Academic Tenure Presented at the Annual Meeting of the Association, December 31, 1915», American Political Science Review vol. 10, $\mathrm{n}^{\circ} 2$ (1916), p. 20.

471915 Declaration of Principles on Academic Freedom.

48 BARENDT, E., op. cit., p. 168. 
reitera además la libertad para discutir en clase las materias propias de su área, y se vuelve a consagrar finalmente la libertad de expresión y de acción fuera de las universidades ${ }^{49}$.

El valor jurídico de estos principios de la AAUP ha sido calificado como soft law, porque no son directamente aplicados por los tribunales ${ }^{50}$. Como contrapartida, un gran número de instituciones educativas los suscribe, y muchos de ellos son incorporados en los contratos de los profesores que ingresan. Los Tribunales de Justicia suelen mencionarlos a propósito de los incumplimientos de contrato ${ }^{51}$.

De la lectura de la Declaración de 1915, del 1940 Statement of Principles of Academic Freedom and Tenure, y de los Comentarios Interpretativos de 1970, parecen iluminarse las siguientes conclusiones:

El problema de la tensión entre la religión y esta idea de libertad de cátedra aparece a propósito de la distinción que se formula entre instituciones privadas y no privadas (que debe entenderse en términos de interés privado y no público, de acuerdo con la distinción realizada antes). La «universidad privada» es aquí fundamentalmente la universidad confesional, es decir, la que establece un vínculo institucional entre ciencia y fe. El punto central es que los redactores de la Declaration de 1915 advierten que los Consejos de las -así llamadas- instituciones privadas actúan de modo diferente de las universida-

49 1. Los profesores tienen derecho a una total libertad en el desarrollo de sus investigaciones y en las publicaciones de sus resultados, sujetos a un adecuado desempeño de sus otros deberes académicos. La investigación remunerada, sin embargo, debiera estar basada en un acuerdo con las autoridades de la institución.

2. Los profesores son libres, en la sala de clases, para discutir sus materias, pero deben ser cuidadosos de no introducir en sus clases cuestiones controvertidas que no tengan relación con éstas. Con respecto a las limitaciones a la libertad de cátedra por causa de objetivos religiosos u otros, la institución debe dejarlas claramente establecidas por escrito a la hora de la contratación. 3. Los profesores de los colleges y las universidades son ciudadanos, integrantes de una profesión ilustrada, y empleados de una institución educativa. Cuando hablan o escriben en calidad de ciudadanos, deben estar libres de la censura o la disciplina institucional, pero su especial posición en la comunidad les impone obligaciones especiales. Como académicos y empleados educativos, debieran recordar que el público puede juzgar su profesión y su institución por sus dichos. Por tanto, deben ser responsables en toda ocasión, deben ejercer control [sobre sí mismos], y ser respetuosos con las opiniones de otros, así como hacer notar que hablan a título personal. 1940 Statement of Principles on Academic Freedom and Tenure. Academic Freeedom. La traducción es nuestra.

50 Alstyne, W. van, «Academic Freedom and First Amendment in the Supreme Court of the United States: An Unhurried Historical Review», Law and Contemporary Problems, vol. 53 (1990), p. 79.

51 Cfr. Krotkoff v Goucher College 585 F 2d 675 (1978), cit. por BARENDT, E., op. cit., p. 172. 
des del Estado, por cuanto estas últimas estarían comprometidas con el conocimiento, mientras que las otras obrarían «según los que sean sus puntos de vista», usando el College como «instrumento de propaganda» de ellos ${ }^{52}$, es decir, como fuente de difusión de sus principios religiosos o de otra especie. Se considera que tales instituciones privadas no aceptan los principios de libertad de investigación, de opinión ni de enseñanza; siendo su propósito no el avance del conocimiento por la investigación sin restricciones y la discusión libre por académicos imparciales, sino más bien apoyar la difusión de opiniones emitidas por sujetos normalmente no académicos, que proporcionan los fondos de la institución ${ }^{53}$. Hay aquí una serie de presupuestos que los redactores de la Declaración no se sienten llamados a demostrar, probablemente porque expresaban los presupuestos metodológicos dominantes en la comunidad académica norteamericana. Estas afirmaciones son dos: (a) el objeto de las instituciones de educación superior es esencialmente hacer avanzar el conocimiento, y (b) para ello el concepto central es la libertad.

En lo que se refiere a la primera afirmación (a), se advierte la influencia del concepto de «universidad de investigación» desarrollado por la doctrina alemana, cuya presencia reafirma de paso la búsqueda de profesionalización de la actividad académica. De acuerdo con el modelo alemán, la universidad debe ser semejante a un sistema de total racionalidad, cuyo objetivo es el conocimiento como fin en sí mismo, y secundariamente como elemento de servicio a la sociedad. Mientras que en la universidad escolástica el conocimiento de la realidad se entendía un medio para alcanzar la perfección, cuyo contenido era preeminentemente moral y abierto a la trascendencia, en la universidad moderna, por su énfasis en el método más que en los contenidos, el conocimiento en cuanto tal se desplaza de posición y ocupa el lugar del fin. Esta concepción se transmite a la universidad de investigación norteamericana ${ }^{54}$.

Sobre el segundo presupuesto (b), cabe apuntar que, según este modelo, la libertad no es sólo el medio a través del cual se alcanza el objetivo del conoci-

$52 \ll \mathrm{Si}$ una Iglesia o congregación religiosa funda un college para ser gobernado por un consejo de administración (board of trustees), con el acuerdo expreso de que dicho college será utilizado como instrumento de propaganda de los intereses de la fe profesada por la Iglesia o congregación que la crea, los consejeros tienen el derecho a exigir que todo se subordine a ese fin». 1915 Declaration of Principles on Academic Freedom. 1. Basis of Academic Authority.

531915 Declaration of Principles on Academic Freedom. 1.Basis of Academic Authority.

54 Sobre la universidad de investigación norteamericana, véase el número especial de la revista Daedalus, vol. 122, nº 4 (Otoño 1993). 
miento, sino que constituye en realidad el primer y máximo bien del académico. Su primacía supone, como se decía antes, un giro antropológico. Dado el valor preeminente del método en el proceso cognoscitivo, la libertad pasa a ser la primera e indiscutible exigencia de su ejecución. Esta es la razón de fondo por la que la universidad moderna pone el énfasis en los ámbitos de autonomía del profesor, y por ello el concepto de libertad de cátedra es esencialmente moderno.

De este modo, resulta coherente negar a las instituciones que ponen en cuestión tales presupuestos el valor de un estatuto epistemológico sustentable, afirmando que su objeto es sólo el proselitismo, no el conocimiento racional o intersubjetivo, que sería patrimonio exclusivo de su paradigma epistemológico puramente empírico. Así lo confiesa expresamente Dewey, al afirmar que al discutir las cuestiones que se resumen en la expresión 'libertad de cátedra', es necesario distinguir entre las universidades propiamente dichas, y aquellos cuerpos docentes cuyo objetivo primario es inculcar un conjunto determinado de hechos e ideas. Las primeras buscarían descubrir y comunicar la verdad, y hacer de la audiencia mejores jueces de la verdad y mejores aplicadores de ella en los asuntos cotidianos. Los segundos tendrían por objetivo el perpetuar una cierta manera de ver las cosas de un grupo de personas ${ }^{55}$. Dewey no niega el derecho de cualquier institución religiosa, política o incluso económica de regentar centros de educación superior desde un punto de vista jurídico ${ }^{56}$; su argumento $-\mathrm{y}$ el de la AAUP- es más bien de naturaleza moral: tales instituciones no podrían alcanzar la verdad, que les estaría vedada por el hecho de sustentar determinadas proposiciones que son consideradas ex ante como verdaderas. Serían, por decirlo así, creaciones esperpénticas, pero lícitas, en base justamente a la libertad que, como un trasfondo, constituiría el verdadero horizonte de significado de la acción humana.

De esta manera, mediante el recurso de sustentar implícitamente un modelo metodológico (el moderno) con preferencia sobre el otro (el escolástico), y considerarlo el único posible, se realiza un sutil movimiento de piezas que excluye del universo discursivo -y por lo tanto de la noción de libertad de cátedra- a un grupo de instituciones universitarias reales y concretas, no sólo de la sociedad norteamericana, sino emparentadas algunas de ellas (las de confesión o de inspiración religiosa) con la tradición occidental que, irónicamente, es la que da origen al concepto mismo de universidad a partir del siglo XI.

55 DewEY, J., «Academic Freedom», Educational Review, vol. XXIII (enero-mayo 1902), p. 1.

56 Ibid., pp. 1-2. 
El principio subyacente a la Declaración de 1915 es que tales instituciones tienen un interés privado, no público, es decir, su objeto no es el conocimiento -con el cual toda la sociedad está comprometida, para su beneficio-, sino otros fines distintos, cualquiera que ellos sean. Resurge aquí la tensión entre lo público y lo privado como una configuración propia de la modernidad, que para la universidad medieval tenía una línea de distinción mucho más tenue, como se indicaba antes. Y por lo tanto, dichos centros no deben ser financiados ni tienen potestad moral para limitar la libertad de los académicos ${ }^{57}$. Así, toda universidad que no comparta el modelo de una libertad de cátedra sin restricciones, no busca en realidad el conocimiento, sino el partisanismo y la propaganda, y en consecuencia carece de valor público. Al no disponer de valor público, no debe recibir financiamiento del Estado, ni será legítima la generación de modalidad alguna que limite la autodeterminación de los académicos en razón de sus objetivos institucionales, en caso de que dicha indicación entre en conflicto con el paradigma de la libertad académica promulgado por la AAUP. El único modelo universitario dotado de valor moral y jurídico, por lo tanto, resulta ser el que realice tales postulados metodológicos, lo que le dotaría de un valor general, no privado, que aconseja su preservación.

La Declaración de 1915 afirma que el Comité no se va a pronunciar sobre si es o no deseable que existan tales instituciones, pero le parece de primera importancia que no se vistan con falsos ropajes (es decir, que no pretendan hacerse pasar por universidades en sentido «público-moral»). Lo anterior hace suponer que, en esta lógica, tarde o temprano debieran ser despojadas de su denominación propiamente universitaria, por cuanto no les corresponde en realidad, si persisten en su actitud. El texto se felicita de que este tipo de institución sea cada vez más infrecuente en Estados Unidos, porque cada vez se encuentran de hecho más alejadas de sus principios fundacionales, lo que es visto como una transición saludable ${ }^{58}$. Algo similar se puede leer en Dewey, cuando sostiene que muchas instituciones se encuentran en un estado de transición,

571915 Declaration of Principles on Academic Freedom. 1. Basis of Academic Authority.

58 «Tales instituciones son una excepción y se están convirtiendo en cada vez más excepcionales. De hecho, todavía tenemos colleges bajo denominación confesional, pero muy poco imponen a sus miembros la obligación de difundir doctrinas específicas. Ocupan cada vez más, en relación con la libertad de los miembros del claustro, el lugar de instituciones carentes de restricciones se están convirtiendo, cada vez más, en más excepcionales. De hecho, todavía tenemos colleges confesionales; cuya diferencia se manifiesta sólo en base a sus antecedentes y tradiciones históricas». 1915 Declaration of Principles on Academic Freedom. 1. Basis of Academic Authority. 
por cuanto, desde el punto de vista histórico, estarían atadas a un conjunto de creencias, a las cuales nominalmente todavía les deben una cierta lealtad; sin embargo estarían simultáneamente asumiendo funciones de universidad en sentido estricto, y aceptando en consecuencia obligaciones con toda la sociedad (y no sólo una parte de ella) ${ }^{59}$. A mayor abundamiento, el tercer comentario de 1970 sobre el Statement de 1940 sostiene que la mayor parte de las instituciones ligadas a iglesias en Estados Unidos ya no deseaban ni buscaban diferir del principio de libertad de cátedra contenido en el texto de 1940.

\section{Algunas CONSIDERACiONES FINALES}

Como puede observarse a partir de lo explicado hasta ahora, la cuestión debatida entre el conocimiento por fe y el conocimiento científico obedece, en su dimensión más íntima, a una diferencia en la concepción antropológica que se halla detrás de cada modelo. De un modo más concreto: el papel que cabe al conocimiento por fe en la vida académica dependerá en última instancia del valor que se le asigne para constituir y reflejar la realidad cognitiva humana en su integridad. La universidad de la escolástica (y las universidades confesionales de nuestro tiempo) consideraba dotadas de valor científico todas las formas de conocimiento que le acontecen al ser humano: conocimiento sensible, racional y revelado. Esto era así porque en su precomprensión del mundo y del hombre se encontraba la intuición de que la realidad era esencialmente unitaria, y que los fragmentos de verdad no podían estar aislados ni ser contradictorios.

Para la universidad moderna en cambio, que desarticula el valor científico de las proposiciones situadas más allá de la verificación, las definiciones de la fe -al exceder el ámbito lógico-empírico- no pueden tener valor alguno en perspectiva académica. Por lo tanto, sitúa su influencia en una dimensión pre-científica, obsesionada por la búsqueda de poder mediante la ejecución de actos de proselitismo.

En esta diferencia antropológica que distingue a los dos modelos, resulta crucial la idea de libertad. Para el mundo académico escolástico, la libertad era un atributo por el cual el agente podía hacerse parte en la configuración de sus propios actos, al decidir si actuar conforme o no a la ley natural, y ad-

59 DeweY, J., op. cit., pp. 1-2. 
judicándose por lo tanto el mérito próximo del acto bueno, en función de la bondad o maldad intrínseca de las acciones. En la universidad ilustrada, sin embargo, como se ha hecho problemática la posibilidad de un a priori cognitivo, la conciencia subjetiva avanza hacia el primer plano, otorgando a la idea de libertad un germen de indeterminación en el que dicha conciencia individual se constituye como una especie de núcleo ontológico situado dialécticamente respecto de las realidades exteriores.

La idea moderna de libertad de cátedra responde a esta concepción de la libertad simplemente como ausencia de coacción. Se trata de un derecho concebido al calor de acciones concretas de censura académica, pero que se enmarca dentro de un proceso de traducción al ámbito jurídico de la subjetivización de la conciencia, antes mencionada. Este proceso, en el plano jurídico, se inicia de la mano específicamente de la llamada «Escuela Moderna del Derecho Natural», es decir, Pufendorf, Thomasius y Wolff.

Nos parece, sin embargo, que esta noción de libertad de cátedra -cuyas características se han explicado antes- utiliza una idea reduccionista de libertad, por cuanto se basa en una antropología que a su debido tiempo reduce el espacio de conocimiento propiamente humano a realidades que dejan fuera no sólo los misterios de la fe, sino también los principios metafísicos que constituían el punto de partida del pensar escolástico.

En un sentido inverso, la libertad académica que se fundara en la armonía entre ciencia y fe, representaría en clave institucional, la integridad espiritual humana de un modo mucho más acabado que el derecho creado por la modernidad, por cuanto dicha integridad no puede ser alcanzada sólo a través de la contemplación de las realidades corpóreas, sino que aspira naturalmente a la trascendencia, como expresara brillantemente Santo Tomás al reclamar la imposibilidad de los deseos vanos. La principal consecuencia de este equilibrio entre razón y fe es formal o metodológica, y sólo de un modo secundario material o relativa a los contenidos que se han de conocer. La viabilidad de conocer con certeza entidades que se hallan fuera del campo de lo humano, es condición de posibilidad para declarar, como realidad científicamente viable, al objeto de la metafísica, el ente en cuanto ente y lo que le corresponde de suyo. La existencia encarna ella misma una dimensión misteriosa y primaria del conocimiento, que sólo puede alcanzarse sin embargo de modo periférico, a través de una descripción. Esto no significa que el objeto de la metafísica sea, desde el punto de vista del contenido, una materia de fe. Lo que se quiere decir es que para poder pensar un a priori a todo método, será siempre un requisito conocer por sobre la mera verificación. En este contexto, la libertad de cáte- 
dra, si hubiera existido en el mundo escolástico, sería equivalente a la libertad del scholasticus para alcanzar y comprender, en la medida de sus fuerzas, las realidades que no pueden agotarse bajo la mirada humana, sin verse constreñida por un método específico, propio de un objeto científico, cualquiera que sea, que se proponga como único. La libertad de cátedra se configura así, también, como la libertad para creer en el ámbito científico sin que ello signifique un menoscabo del estatuto del pensamiento, y no indicaría sólo la libertad de coacción académica, como parece entenderse a partir del derecho moderno. En este sentido, la complementariedad de razón y fe parece ser un elemento central para definir en qué sentido es, verdaderamente, libre un académico. 